\title{
Evaluation of Household Cleaning Methods for Reducing Chlorantraniliprole Residues on Cowpea Fruits
}

\author{
Xiao-Jun Chen ${ }^{1}$, Zhi-Yuan Meng ${ }^{1}$, Ping Wang ${ }^{1}$, Chun-Liang Lu ${ }^{2}$, Yi-Zhong Yang ${ }^{1}$, Li Zhang ${ }^{1}$, Li Liu ${ }^{3}$ \\ \& $\mathrm{Si} \mathrm{Chen}^{1}$ \\ ${ }^{1}$ School of Horticulture and Plant Protection, Yangzhou University, Yangzhou, Jiangsu, China \\ ${ }^{2}$ Testing Center of Yangzhou University, Yangzhou University, Yangzhou, Jiangsu, China \\ ${ }^{3}$ College of Food Science and Engineering, Yangzhou University, Yangzhou, Jiangsu, China \\ Correspondence: Xiao-Jun Chen, School of Horticulture and Plant Protection, Yangzhou University, Yangzhou, \\ Jiangsu 225009, China. E-mail: cxj@yzu.edu.cn
}

Received: May 3, 2015 Accepted: June 16, 2015 Online Published: August 15, 2015

doi:10.5539/jas.v7n9p129

URL: http://dx.doi.org/10.5539/jas.v7n9p129

\begin{abstract}
All pesticide residues are toxic by design and pose seriously health dangers to people. We explored an effective cleaning technique of food safety and handling guidelines. Chlorantraniliprole residues on cowpea fruits were determined by LC-MS/MS (liquid chromatography-tandem mass spectrometry) after different cleaning methods. Both soaking the cowpea fruits in water for $15 \mathrm{~min}$ and soaking in $0.1 \%$ edible vinegar for 15 min followed by rinsing with running tap water for 2 min could effectively remove the chlorantraniliprole residues on cowpea fruits. Cleaning with running tap water for 2 min was the worst cleaning method. Both the treatments of soaking in acidic and neutral $\mathrm{pH}$ cleaning solution could generate good removal efficiency of chlorantraniliprole residues on cowpea. Different cleaning solution concentration did not give significant difference removal efficiency. Our research provided the inherent relationship between pesticide residues and cleaning approaches, also the important theoretical basis for risk assessments of food.
\end{abstract}

Keywords: cowpea fruits, chlorantraniliprole, cleaning methods, removal efficiency, processing factor

\section{Introduction}

Pesticide residues on the agricultural produce such as fruits, crops and vegetable are highly regulated today. Although some residues may remain at harvest, residues tend to decline as the pesticide breaks down. Only the pesticide residues is monitored but the cleaning process or processed agricultural products or foods are ignored so far. Most agricultural products are consumed after cleaning. Recently, new less toxic and highly efficient pesticides have been widely applied in agricultural production. Therefore, monitoring these novel pesticides such as chlorantraniliprole before and after cleaning process is necessary. Although these pesticides have low toxicity and low residual on the agricultural products and may degrade during processing or during storage, some degradation products may be more toxic than the parent compounds (St-Amand \& Girard, 2004; Luís et al., 2005; Muhammad et al., 2006; Chai et al., 2008). Therefore, an appropriate cleaning method to remove the residue on produce is important in both household and factory processing to reduce the potential human health risk.

Cowpea (Vigna unguiculata) is an important leguminous vegetable consumed daily in China. It is the host of thrips, pod borer, cutworm, aphids, leaf miner and other major pests (Pan et al., 2005). Inevitably, chemical pesticides are used during the growth process ( $\mathrm{Li}$ et al., 2010). In this study, pesticide residues chlorantraniliprole on the cowpea fruits were cleaned with different techniques following vegetable cleaning habits of Chinese families, namely cleaning including rinsing with running water, cleaning after soaking in water, edible vinegar, edible salt, sodium bicarbonate solution or fruit and vegetable cleaning solution. The aim of this study was to establish the most effective traditional cleaning method for removing chlorantraniliprole residues on the cowpea fruits.

A systematic study of removal efficiency is important for establishing guidelines in food safety and handling that minimize human exposure to pesticide through food. Furthermore, understanding the removal efficiency of different cleaning techniques is a critical step toward characterizing degradation products in the agricultural products during processing, to provide revealed inherent relationships between pesticide residues and processing 
approaches, also provide important theoretical basis for risk assessments of food.

\section{Method}

\subsection{Chemicals and Materials}

Chemservice supplied the technical grade analytical standard of chlorantraniliprole (purity 99.0\%). This formulation (5\% SC) was obtained from Shanghai Dupont Agricultural Chemicals Co., Ltd. Methanol and acetonitrile (HPLC grade) were obtained from American Tedia. Anhydrous magnesium sulfate (AR), N-propyl ethylene diamine (PSA) and C18 were obtained from DIMA Technology Co., Ltd. (USA). Sodium chloride (AR) was obtained from Tianjin Chemical Reagent CO., Ltd. Distilled water was filtered through $0.22 \mu \mathrm{m}$ membrane before using.

Edible vinegar, edible iodine salt, edible sodium bicarbonate, fruits and vegetables cleaning agent were obtained from Hengguan Industry Co., Ltd. Zhenjiang City, Guhuai Salt Co Ltd., Golden Bridge Salt Group, Jiangsu, Honeydew Park Sugar Co Ltd., Nanjing City and Kaimi Corporation Xi'an City, respectively.

\subsection{Preparation of Chlorantraniliprole Residual on Cowpea Fruits}

The cowpeas were grown in Yangzhou University experimental farms.The crisp, full pod, no damage and no mildew cowpea fruits were used as experimental materials. The head and tail sections of the cowpea fruit were removed.

A $100 \mathrm{mg} \mathrm{kg}^{-1}$ soaking solution of chlorantraniliprole (5\% SC) was prepared in deionized water.Cowpea fruits $(10 \mathrm{~kg})$ were completely immersed in $60 \mathrm{~L}$ of the soaking solution for $30 \mathrm{~min}$ at room temperature followed by drying in open air for $1 \mathrm{~h}$.

\subsection{Cleaning Methods}

\subsubsection{Cleaning Solutions}

In treatment one $\left(T_{1}\right)$, the whole cowpea fruits were cleaned with running tap water for 2 min using a sieve to ensure the cowpea fruits were cleaned uniformly during the whole cleaning process. The cowpea fruit samples were then air-dried in a fume hood at room temperature. In the second treatment $\left(\mathrm{T}_{2}\right)$, the whole cowpea fruits were not cleaned. Three replicates were applied for each treatment.

The effects of soaking using different solutions including water, edible vinegar $(0.1 \%)$, edible salt solution $(0.1 \%)$, edible sodium bicarbonate solution $(0.1 \%)$ and fruit and vegetable cleaning solution $(0.1 \%)$ were investigated. Cowpea fruits $(1 \mathrm{~kg})$ were soaked in $4 \mathrm{~L}$ soaking solution (water, edible vinegar $(0.1 \%)$ and edible salt solution $(0.1 \%)$, edible sodium bicarbonate solution $(0.1 \%)$ and fruit and vegetable cleaning solution $(0.1 \%))$ for $5,10,15,30,45$ and $60 \mathrm{~min}$. Cowpea fruits used for the control treatments were not cleaned. The cowpea fruits were rinsed afterward using running tap water for $2 \mathrm{~min}$. Each treatment was repeated three times. The treated samples were air-dried in a fume hood. Chlorantraniliprole on cowpea fruits were extracted, cleaned-up and analyzed using LC-MS/MS. Removal efficiency and processing factor of chlorantraniliprole were calculated according to the residue content of chlorantraniliprole on cowpea fruits.

\subsubsection{Effects of Concentrations of Cleaning Solution}

Soaking solutions of edible vinegar, edible iodine salt, edible sodium bicarbonate and fruits and vegetables cleaning agent were prepared respectively, and the concentrations of each solution contained $0.1 \%, 0.5 \%, 1.0 \%$, $5.0 \%$ and $10.0 \%$. The $\mathrm{pH}$ values of each cleaning solution were measured by Sartorius PB-21 pH standard meter and shown in Table 1. Cowpea fruits $(1 \mathrm{~kg})$ were soaked in $4 \mathrm{~L}$ soaking solution water, edible vinegar $(0.1 \%$, $0.5 \%, 1.0 \%, 5.0 \%$ and $10.0 \%$ ), edible salt solution $(0.1 \%, 0.5 \%, 1.0 \%, 5.0 \%$ and $10.0 \%)$, edible sodium bicarbonate solution $(0.1 \%, 0.5 \%, 1.0 \%, 5.0 \%$ and $10.0 \%)$ and fruit and vegetable cleaning solution $(0.1 \%, 0.5 \%$, $1.0 \%, 5.0 \%$ and $10.0 \%$ ) for 15 min separately. $\mathrm{pH}$ values were measured by Sartorius PB-21 $\mathrm{pH}$ standard meter (Sartorius, German). 
Table 1. pH values of different cleaning solutions

\begin{tabular}{llll}
\hline Cleaning solution & $\mathrm{pH}$ value & Cleaning solution & $\mathrm{pH}$ value \\
\hline $0.1 \%$ edible vinegar solution & 6.18 & $0.1 \%$ edible sodium bicarbonate solution & 8.05 \\
$0.5 \%$ edible vinegar solution & 5.33 & $0.5 \%$ edible sodium bicarbonate solution & 8.05 \\
$1.0 \%$ edible vinegar solution & 4.92 & $1.0 \%$ edible sodium bicarbonate solution & 8.02 \\
$5.0 \%$ edible vinegar solution & 3.15 & $5.0 \%$ edible sodium bicarbonate solution & 7.85 \\
$10 \%$ edible vinegar solution & 2.92 & $10 \%$ edible sodium bicarbonate solution & 7.79 \\
$0.1 \%$ edible salt solution & 5.40 & $0.1 \%$ fruit and vegetable cleaning solution solution solution & 7.55 \\
$0.5 \%$ edible salt solution & 5.71 & $0.5 \%$ fruit and vegetable cleaning solution & 7.50 \\
$1.0 \%$ edible salt solution & 5.89 & $1.0 \%$ fruit and vegetable cleaning solution & 7.42 \\
$5.0 \%$ edible salt solution & 6.48 & $5.0 \%$ fruit and vegetable cleaning solution & 7.24 \\
$10 \%$ edible salt solution & 7.42 & $10 \%$ fruit and vegetable cleaning solution & 7.13 \\
Tap water & 7.31 & & \\
\hline
\end{tabular}

Subsequently, the cowpea fruits were cleaned using running tap water for 2 min only. The control treatments were not cleaned. Three replicates were applied for each treatment. All treated samples were air-dried in a fume hood. Chlorantraniliprole was extracted, cleaned-up and analyzed using LC-MS/MS (liquid chromatography-tandem mass spectrometry). Removal efficiency and processing factor of chlorantraniliprole were calculated according to the residue content of chlorantraniliprole on the cowpea fruits.

\subsection{Extraction of Chlorantraniliprole Residue from Cowpea Fruits}

Cowpea fruits $(5 \mathrm{~g})$ were homogenized in $30 \mathrm{~mL}$ of acetonitrile using a high-speed homogenizer for $3 \mathrm{~min}$. For recovery experiment, chlorantraniliprole was added to the samples. The final concentrations of chlorantraniliprole were 5.00, 1.00, and $0.20 \mu \mathrm{g} \mathrm{g} \mathrm{g}^{-1}$, respectively. A blank control group was also set up, and all experiments were repeated three times.

Chlorantraniliprole was extracted from cowpea fruits using QuEChERS method after different cleaning treatments. Sodium chloride $(1.5 \mathrm{~g})$ and anhydrous magnesium sulfate $(6 \mathrm{~g})$ were added to the samples before centrifuging at $4000 \mathrm{rpm}$ for $5 \mathrm{~min}$. About $2 \mathrm{~mL}$ of the supernatant was collected, and added to the centrifuge tubes containing $\mathrm{MgSO}_{4}(150 \mathrm{mg})$, PSA $(25 \mathrm{mg})$, and $\mathrm{C}_{18}(25 \mathrm{mg})$, and then centrifuged for $5 \mathrm{~min}$. The supernatant $(0.60 \mathrm{~mL})$ was diluted to $1.0 \mathrm{~mL}$ of distilled water. The sample solution was filtered through a 0.22 $\mu \mathrm{m}$ membrane and analyzed on LC-MS/MS.

\subsection{Instrumental Analysis}

Samples were weighed by BS210S electronic balance (Sartorius, Germany), concentrated by SB-1000 rotary vacuum evaporator (Eyela, Japan) and mixed using a THZ-82A vibrating machine (FuHua, Jiangsu Province, China). Separation and detection were carried out using Agilent 1200 HPLC system (Agilent Co., USA) equipped with a binary pump, auto plate-sampler, column oven, and diode-array detector. Mass spectra were recorded on an Agilent 6460 triple quadrupole (QQQ) mass spectrometer equipped with an ESI source. The quantification of chlorantraniliprole residues were determinded by LC-MS/MS as described by Chen (Chen et al., 2013).

\subsection{Calculated Methods of Removal Rate and Processing Factor}

Removal rate and processing factor of each treatment were calculated. Formulas of removal rate and processing factor were as below.

$$
\begin{gathered}
\text { Removal rate }(\%)=\frac{\text { Initial concentration }- \text { Residues concentration }}{\text { Initial concentration }} \times 100 \\
\text { Processing factor }=\frac{\text { Residues concentrat ion }}{\text { Initial concentrat ion }}
\end{gathered}
$$

All data were analyzed by analysis of Duncan multiple comparison. All the experiments were repeated three as means \pm standard error of mean. Different lowercase letters after the number was $5 \%$ significant difference and different uppercase letters after the number was $1 \%$ significant difference. 


\section{Results}

\subsection{Fortified Recoveries of Chlorantraniliprole from the Cowpea Fruits}

A standard stock solution of chlorantraniliprole $\left(1 \mathrm{mg} \mathrm{mL}^{-1}\right)$ was prepared in HPLC grade acetonitrile. The standard solutions required for constructing a calibration curve $\left(50.0,25.0,12.5,6.25,3.125 \mu \mathrm{g} \mathrm{mL}^{-1}\right)$ were prepared from stock solution by serial dilution with HPLC grade acetonitrile. All standard solutions were stored at $4{ }^{\circ} \mathrm{C}$ before using. The quantification of chlorantraniliprole residues uses an external standard.

To know the efficiency of extraction and clean-up procedures, recovery experiments were carried out at different levels to establish the reliability and validity of analytical method. The control samples of cowpea fruits were spiked at $5,1.0$, and $0.2 \mathrm{mg} \mathrm{kg}^{-1}$ respectively followed by methodology as described above. The average recoverie was above $80 \%$ (Table 2 ). The coefficients of variation were $1.22 \%$ to $4.12 \%$ and $2.10 \%$ to $4.90 \%$, respectively.

Table 2. The recoveries of chlorantraniliprole from the cowpea fruits

\begin{tabular}{llll}
\hline Fortification levels $\left(\mathrm{mg} \mathrm{kg}^{-1}\right)$ & Average recoveries (\%) & Standard deviation & Coefficient of variance (\%) \\
\hline 5.0 & 92.80 & 0.1005 & 1.18 \\
1.0 & 90.33 & 0.0103 & 2.23 \\
0.2 & 81.53 & 0.0068 & 4.28 \\
\hline
\end{tabular}

Note. The remaining amount was the average of three replicates.

\subsection{Removal Efficiency of the Cleaning Using Running Tap Water}

Removal rate was $12.79 \%$ and processing factor was 0.8721 when the cowpea fruit residue samples were cleaned under a simple cleaning with running tap water for $2 \mathrm{~min}$ and its removal rate was low (Table 3 ).

Table 3. Removal effects of residue chlorantraniliprole on the cowpea fruits soaked in the tap water followed by cleaning with running tap water

\begin{tabular}{lllll}
\hline Treatment method & Initial concentration $\left(\mathrm{mg} \mathrm{kg}^{-1}\right)$ & Residues concentration $(\mathrm{mg} / \mathrm{kg})$ & Removal rate $(\%)$ & Processing factor \\
\hline Tap water & $9.2930 \pm 0.0583$ & $8.1048 \pm 0.5433$ & $12.79 \pm 5.8457$ & $0.8721 \pm 0.0585$ \\
\hline
\end{tabular}

Note. The experiments were set up in a completely randomized design. All the experiments were repeated thrice as means \pm standard error of mean.

Removal rate was $68.42 \%$ and processing factors was 0.3158 when the samples were soaked in the water for 15 min and cleaned with running tap water for 2 min (Table 4). The removal rates of the cowpea fruits soaked in the water for different time followed by cleaning with running tap water for 2 min were higher than those of the cowpea fruits cleaned with running tap water for 2 min only. The removal rate was not changed significantly even with the extension of soaking time in water.

Table 4. Removal effects of the cowpea fruits soaked in tap water followed by cleaning with running tap water

\begin{tabular}{lllll}
\hline Soaking solution & Initial concentration $\left(\mathrm{mg} \mathrm{kg}^{-1}\right)$ & Residues concentration $(\mathrm{mg} / \mathrm{kg})$ & Removal rate (\%) & Processing factor \\
\hline tap water & $8.3636 \pm 0.2872$ & $2.6638 \pm 0.7263$ & $68.42 \pm 8.680$ & $0.3158 \pm 0.0868$
\end{tabular}

Note. The experiments were set up in a completely randomized design. All the experiments were repeated thrice as means \pm standard error of mean.

\subsection{Removal Efficiency of Different Cleaning Methods on Removing the Residue Pesticide on the Cowpea Fruits}

Removal efficiency of different cleaning methods on removing the residue pesticide on the cowpea fruits: removal rate were $25.87 \% \sim 80.57 \%$ and processing factors were $0.1970 \sim 0.7413$ when the cowpea fruit soaked in water for different times followed by cleaning with running tap water for $2 \mathrm{~min}$ (Figure 1). The removal rates of 
the cowpea fruits soaked in water for different time followed by cleaning with running tap water for 2 min were higher than those of the cowpea fruits cleaned with running tap water for 2 min only. The removal rate was not changed significantly with the extension of soaking time in solution. Removal rates were $24.97 \% \sim 78.86 \%$ and processing factors were $0.2114 \sim 0.7503$ when the cowpea fruit residue samples soaked in the edible vinegar solution for different time followed by cleaning with running tap water for 2 min (Figures 1-2). Under the extension of the soaked time in the $0.1 \%$ edible vinegar solution conditions, removal rate was not changed obviously over soaked for $15 \mathrm{~min}$.

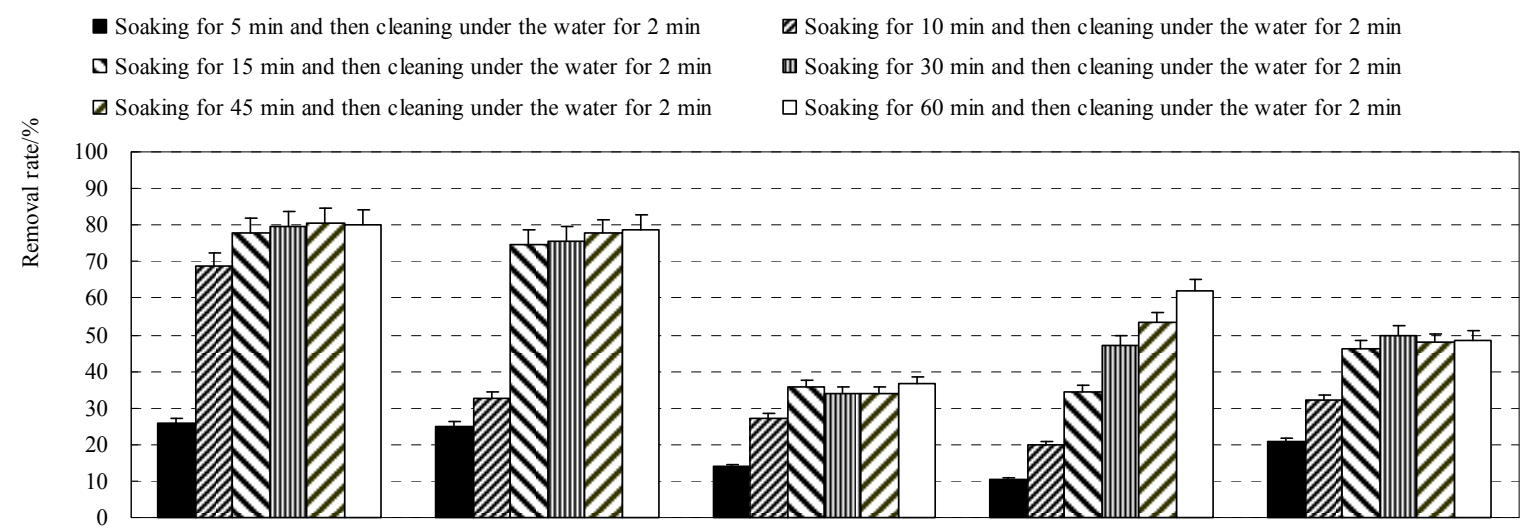

Figure 1. Removal rates of residue chlorantraniliprole on the cowpea fruits soaked in different solutions for different times

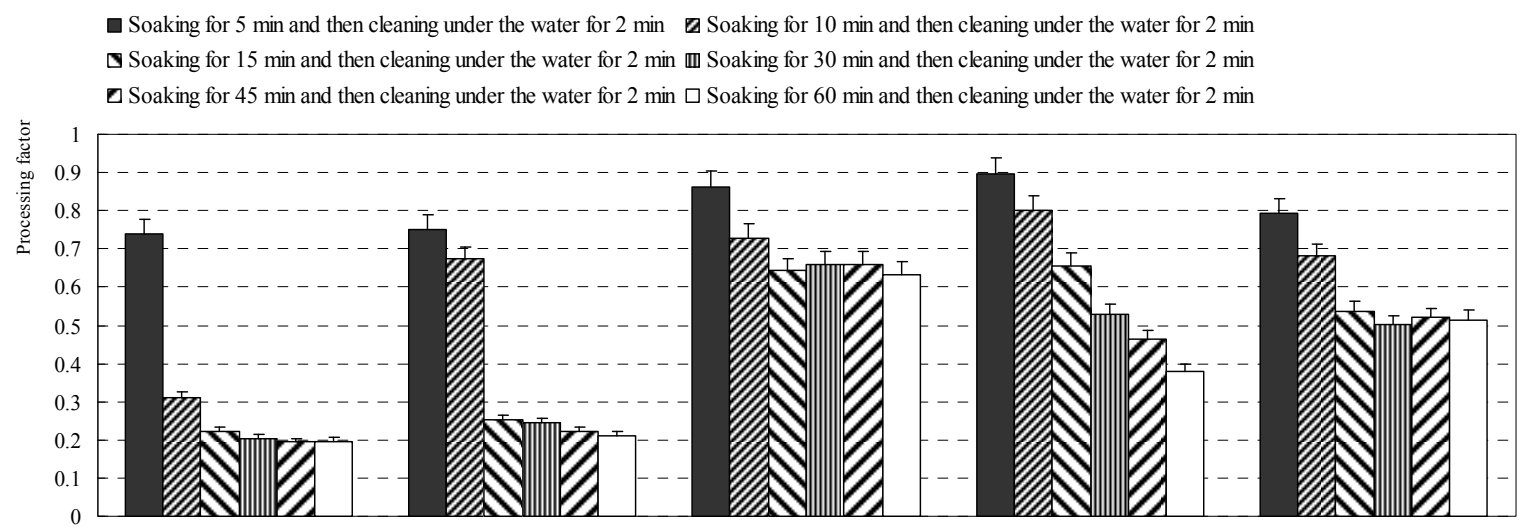

Figure 2. Processing factors of residue chlorantraniliprole on the cowpea fruits soaked in different solutions for different times

Our results indicated that cleaning methods including soaking in the water followed by cleaning with running tap water and soaking in $0.1 \%$ edible vinegar followed by cleaning with running tap water were the preferred treatment methods. The removal rate was $12.79 \%$ and processing factor being 0.8721 when the cowpea fruits were cleaned with running tap water only. It was the worst cleaning method.

\subsection{Results of the Cowpea Fruits Soaked in Different Concentrations Solution Followed by Cleaning with Running Tap Water}

3.4.1 Removal Efficiency of the Cowpea Fruits Soaked in Different Concentrations of Edible Vinegar Solution Followed by Cleaning with Running Tap Water

The mean initial concentration was $8.3640 \mathrm{mg} \mathrm{kg}^{-1}$ as the concentration of above processing samples. The final concentration was as the concentration of the samples that were soaked in different concentrations of edible vinegar followed by cleaning with running tap water for $2 \mathrm{~min}$. Removal rate were $56.71 \% \sim 67.03 \%$ and processing factors were $0.3204 \sim 0.4329$ (Table 5). With the increasing concentration of edible vinegar solution, removal rate was decreased slightly only. 
Table 5. Removal effects of the cowpea fruits soaked in different concentrations of edible vinegar solutions followed by cleaning with running tap water

\begin{tabular}{lllll}
\hline Soaking solution & Initial concentration $\left(\mathrm{mg} \mathrm{kg}^{-1}\right)$ & Residues concentration $\left(\mathrm{mg} \mathrm{kg}^{-1}\right)$ & Removal rate $(\%)$ & Processing factor \\
\hline $0.1 \%$ vinegar solution & $8.3640 \pm 0.2844$ & $2.7575 \pm 0.2857$ & $67.03 \pm 5.3710 \mathrm{Aa}$ & $0.3204 \pm 0.0537 \mathrm{Aa}$ \\
$0.5 \%$ vinegar solution & $8.3640 \pm 0.2844$ & $3.0750 \pm 0.2623$ & $63.27 \pm 5.0925 \mathrm{Aa}$ & $0.3673 \pm 0.0506 \mathrm{Aa}$ \\
$1.0 \%$ vinegar solution & $8.3640 \pm 0.2844$ & $3.4010 \pm 0.4481$ & $59.34 \pm 5.3549 \mathrm{Aa}$ & $0.4066 \pm 0.0535 \mathrm{Aa}$ \\
$5.0 \%$ vinegar solution & $8.3640 \pm 0.2844$ & $3.4627 \pm 0.3874$ & $58.60 \pm 4.6304 \mathrm{Aa}$ & $0.4140 \pm 0.0535 \mathrm{Aa}$ \\
$10 \%$ vinegar solution & $8.3640 \pm 0.2844$ & $3.6204 \pm 0.3115$ & $56.71 \pm 5.6821 \mathrm{Aa}$ & $0.4329 \pm 0.0568 \mathrm{Aa}$ \\
\hline
\end{tabular}

Note. The experiments were set up in a completely randomized design. All data were analyzed by Duncan multiple comparison. All the experiments were repeated thrice as means \pm standard error of mean. Different lowercase letters after the number was $5 \%$ significant difference and different uppercase letters after the number was $1 \%$ significant difference. Notes of Tables 5-7 were same to Table 4 .

3.4.2 Removal Efficiency of the Cowpea Fruits Soaked in Different Concentrations of Edible Salt Solution Followed by Cleaning with Running Tap Water

The final concentration was as the concentration of the samples that were soaked in different concentrations of edible salt solution followed by cleaning with running tap water for 2 min. Removal rate were $31.70 \% \sim 58.46 \%$ and processing factors were $0.4154 \sim 0.6830$ (Table 6 ).

Table 6. Removal effects of the cowpea fruits soaked in the different concentrations of salt solution followed by cleaning with running tap water

\begin{tabular}{lllll}
\hline Soaking solution & Initial concentration $\left(\mathrm{mg} \mathrm{kg}^{-1}\right)$ & Residues concentration $\left(\mathrm{mg} \mathrm{kg}^{-1}\right)$ & Removal rate $(\%)$ & Processing factor \\
\hline $0.1 \%$ salt solution & $8.4111 \pm 0.8209$ & $5.3946 \pm 0.8636$ & $35.86 \pm 2.1587 \mathrm{Bb}$ & $0.6414 \pm 0.0216 \mathrm{Bb}$ \\
$0.5 \%$ salt solution & $8.4111 \pm 0.8209$ & $5.7442 \pm 0.4282$ & $31.70 \pm 5.0930 \mathrm{Bb}$ & $0.6830 \pm 0.0509 \mathrm{Bb}$ \\
$1.0 \%$ salt solution & $8.4111 \pm 0.8209$ & $5.2741 \pm 0.4646$ & $37.29 \pm 9.3019 \mathrm{Bb}$ & $0.6271 \pm 0.0930 \mathrm{Bb}$ \\
$5.0 \%$ salt solution & $8.4111 \pm 0.8209$ & $4.8185 \pm 2.4528$ & $42.71 \pm 9.1594 \mathrm{Bb}$ & $0.5729 \pm 0.0916 \mathrm{Bb}$ \\
$10 \%$ salt solution & $8.4111 \pm 0.8209$ & $3.4943 \pm 0.5315$ & $58.46 \pm 6.3216 \mathrm{Aa}$ & $0.4154 \pm 0.0632 \mathrm{Aa}$ \\
\hline
\end{tabular}

3.4.3 Removal Efficiency of the Cowpea Fruits Soaked in Different Concentrations of Edible Sodium Bicarbonate Solution Followed by Cleaning with Running Tap Water

The final concentration was as the concentration of the samples that were soaked in different concentrations of sodium bicarbonate solution followed by cleaning with running tap water for $2 \mathrm{~min}$. Removal rate were $49.78 \% \sim 63.28 \%$ and processing factors were $0.3672 \sim 0.5022$ (Table 7 ).

Table 7. Removal effects of the cowpea fruits soaked in the different concentrations of sodium bicarbonate solution followed by cleaning with running tap water

\begin{tabular}{lllll}
\hline Soaking solution & Initial concentration $\left(\mathrm{mg} \mathrm{kg}^{-1}\right)$ & Residues concentration $\left(\mathrm{mg} \mathrm{kg}^{-1}\right)$ & Removal rate $(\%)$ & Processing factor \\
\hline $0.1 \% \mathrm{NaHCO}_{3}$ solution & $8.3636 \pm 0.2827$ & $4.2002 \pm 0.2905$ & $49.78 \pm 5.4260 \mathrm{Aa}$ & $0.5022 \pm 0.1543 \mathrm{Aa}$ \\
$0.5 \% \mathrm{NaHCO}_{3}$ solution & $8.3636 \pm 0.2827$ & $3.6359 \pm 0.4244$ & $56.52 \pm 5.0737 \mathrm{Aa}$ & $0.4348 \pm 0.0507 \mathrm{Aa}$ \\
$1.0 \% \mathrm{NaHCO}_{3}$ solution & $8.3636 \pm 0.2827$ & $3.7559 \pm 0.3772$ & $55.09 \pm 4.5092 \mathrm{Aa}$ & $0.4491 \pm 0.0451 \mathrm{Aa}$ \\
$5.0 \% \mathrm{NaHCO}_{3}$ solution & $8.3636 \pm 0.2827$ & $3.3013 \pm 1.0701$ & $60.53 \pm 2.7942 \mathrm{Aa}$ & $0.3947 \pm 0.0279 \mathrm{Aa}$ \\
$10 \% \mathrm{NaHCO}_{3}$ solution & $8.3636 \pm 0.2827$ & $3.0712 \pm 0.8009$ & $63.28 \pm 9.5784 \mathrm{Aa}$ & $0.3672 \pm 0.0958 \mathrm{Aa}$ \\
\hline
\end{tabular}

3.4.4 Removal Efficiency of the Cowpea Fruits Soaked in Different Concentrations of Fruit and Vegetable Cleaning Solution Followed by Cleaning with Running Tap Water

The final concentration was as the concentration of the samples that were soaked in different concentrations of fruit and vegetable cleaning solution followed by cleaning with running tap water for $2 \mathrm{~min}$. Removal rate were 
$31.93 \% \sim 41.27 \%$ and processing factors were $0.5873 \sim 0.6807$ (Table 8 ).

Table 8. Removal effects of the cowpea fruits soaked in the different concentrations of fruit and vegetable cleaning solution followed by cleaning with running tap water

\begin{tabular}{lllll}
\hline Soaking solution & $\begin{array}{l}\text { Initial concentration } \\
\left(\mathrm{mg} \mathrm{kg}^{-1}\right)\end{array}$ & $\begin{array}{l}\text { Residues concentration } \\
\left(\mathrm{mg} \mathrm{kg}^{-1}\right)\end{array}$ & $\begin{array}{l}\text { Removal rate } \\
(\%)\end{array}$ & Processing factor \\
\hline $0.1 \%$ fruit and vegetable cleaning solution & $8.2391 \pm 0.2443$ & $4.8392 \pm 0.7072$ & $41.27 \pm 2.7153 \mathrm{Aa}$ & $0.5873 \pm 0.0272 \mathrm{Aa}$ \\
$0.5 \%$ fruit and vegetable cleaning solution & $8.2391 \pm 0.2443$ & $5.0713 \pm 1.2001$ & $38.45 \pm 4.5632 \mathrm{Aa}$ & $0.6155 \pm 0.0456 \mathrm{Aa}$ \\
$1 \%$ fruit and vegetable cleaning solution & $8.2391 \pm 0.2443$ & $5.4108 \pm 0.7851$ & $34.33 \pm 9.5262 \mathrm{Aa}$ & $0.6567 \pm 0.0953 \mathrm{Aa}$ \\
$5 \%$ fruit and vegetable cleaning solution & $8.2391 \pm 0.2443$ & $5.3606 \pm 1.0585$ & $34.94 \pm 2.8441 \mathrm{Aa}$ & $0.6506 \pm 0.0284 \mathrm{Aa}$ \\
$10 \%$ fruit and vegetable cleaning solution & $8.2391 \pm 0.2443$ & $5.6092 \pm 1.1834$ & $31.93 \pm 4.3603 \mathrm{Aa}$ & $0.6807 \pm 0.0436 \mathrm{Aa}$ \\
\hline
\end{tabular}

Our data showed that removal efficiency of chlorantraniliprole residues from the cowpea fruits soaked in acidic or neutral cleaning solution were better. Their removal rates were $64.82 \%$ and $67.03 \%$ when the cowpea fruits soaked in tap water $(\mathrm{pH} \mathrm{7.31)}$ and $0.1 \%$ edible vinegar solution $(\mathrm{pH} \mathrm{6.18)}$ for $15 \mathrm{~min}$, respectively. Different cleaning solution concentration did not lead to significant changing of removal efficiency.

\section{Discussion}

The most critical step in the agro-processing industry is the cleaning process, which is a simple and effective procedure for removing pesticide residues on agricultural products. Removal of food contaminants by cleaning is important in both household and factory processing. The reduction of the levels of pesticide residues on agricultural products is influenced by several factors. The removal rate is affected by the physicochemical properties of the pesticides including octanol/water partition coefficient $\left(\mathrm{K}_{\mathrm{ow}}\right.$ value), solubility and $\mathrm{pKa}$. The physical and chemical characteristics of the cleaning solution, such as cleaning fluid temperature, $\mathrm{pH}$ and polarity also influence the removal rate (Rasmussen et al., 2003; Radwan et al., 2005; Lin et al., 2006; Zhang et al., 2007; Kin et al., 2010; Gonzalez-Rodriguez et al., 2011).

Removal efficiency of residual chlorantraniliprole on the cowpea fruits by the tap water or different concentrations of cleaning solution were discussed. In addition, the $\mathrm{pH}$ value and emulsifying properties and others characters of the cleaning solution had been changed and which could change the pesticide removal effects on the agricultural products because different cleaning agents (edible vinegar, edible salt, edible sodium bicarbonate, fruit and vegetable cleaning agents) were added to the cleaning solution. It indicated that $0.1 \%$ sodium bicarbonate weak alkaline solution had a medium removal ability to remove residue chlorantraniliprole on the cowpea fruits, but it was lower than of $0.1 \%$ edible vinegar solution. It may be explained that chlorantraniliprole has higher solubility in acidic medium or neutral medium and it can access to the cleaning solution more easily when the cowpea fruits were soaked in the acidic solution. Our results were similar to the removal effects of residual methamidophos and rogor in the water solution (Lin et al., 2006) or residual chlorpyrifos on the cabbage (Zhang et al., 2007).

In the cleaning process, the physical and chemical properties of pesticides play an important role in reducing the level of pesticide residues on the agricultural products. Octanol/water partition coefficient $\left(\mathrm{K}_{\text {ow }}\right.$ value $)$ of the pesticide determines the amount of agricultural pesticides on the agricultural products entering into its surface wax layer. The pesticides can more easily enter into the agricultural products' surface layer wax when the $\mathrm{K}_{\text {ow }}$ values are high. The residue pesticides are not easily removed by dissolving cleaning fluid cleaning. Therefore it is difficult to reduce these pesticide residues on the agricultural products. The removal effects of residue pesticides of chlorpyrifos and other 13 different kinds of high $\mathrm{K}_{\mathrm{ow}}$ values on the apples were researched by Rasmussen. Only $52 \%$ of tolylfluanid residues were removed when the apples were cleaned with running tap water for 15 min (Rasmussen et al., 2003). Guardia-Rubio studied the removal effects of residue pesticides such as simazine, diuron and other five kinds of pesticides on olive through the cleaning operation. The results showed that $50 \%$ of the residual simazine could be reduced. But other 4 pesticides reduction effect was not obvious through the cleaning operation. It can explain what $\mathrm{K}_{\mathrm{ow}}$ value of simazine was lower than those of other four kinds of pesticides (Guardia-Rubio et al., 2007). Furthermore, it showed that pesticide residues sprayed on the olive for one day could be cleaned more easily than those sprayed one week before because the surface of the agricultural pesticide had been entered into waxy layer for a longer time and was difficult to be removed through the cleaning (Rasmussen et al., 2003). The removal effects of residue pesticides had a certain relationship with 
the solubility of pesticide in the cleaning solution. Residue pesticides on the cucumbers and strawberries could be cleaned by $10 \%$ acetic acid aqueous. The removal effects were related to the pesticide solubility and vapor pressure. The pesticide could be removed easily with high solubility in the cleaning solution and high vapor pressure during the cleaning process (Kin et al., 2010).

Six cleaning methods based on the vegetables cleaning habits of Chinese families namely, running tap water and soaking in water, $0.1 \%$ edible vinegar solution, edible salt solution, $0.1 \%$ edible sodium bicarbonate solution or $0.1 \%$ fruit and vegetable cleaning solution were selected to remove the residue pesticide chlorantraniliprole on the cowpea fruits. Cleaning methods both soaking in the water and soaking in $0.1 \%$ edible vinegar followed by cleaning with running tap water were the preferred treatment methods. Soaking the cowpea fruits for at least 15 minutes achieved the best removal. The results could provide important theoretical guides of food safety and correct cleaning methods.

\section{Conclusions}

Our results indicated that cleaning methods including soaking in water for 15 and soaking in $0.1 \%$ edible vinegar for $15 \mathrm{~min}$ then cleaning with running tap water for 2 min were the preferred treatment methods. Cleaning with running tap water for 2 min was the worst cleaning method.Removal effects of chlorantraniliprole residues from the cowpea fruits soaked in acidic or neutral $\mathrm{pH}$ cleaning solution were better. For the same cleaning solution, the removal effects of treatments had no significant difference with the changes of cleaning solution concentration.

\section{Acknowledgements}

We thank the Natural Science Foundation of Jiangsu Province (BK20130443) and the Social Development Science and Technology Projects of Yangzhou City, China (2012110) and for providing financial support.

\section{References}

Chai, L. K., Mohd-Tahir, N., \& Hansen, H. C. B. (2008). Determination of chlorpyrifos and acephate in tropical soils and application in dissipation studies. International Journal of Environmental and Analytical Chemistry, 88, 549-560. http://dx.doi.org/10.1080/03067310802002508

Chen, X. J., Cui, H. R., Fan, S. Q., Wang, M., Lu, C. L., \& Yang, Y. Z. (2013). Systemicity of chlorantraniliprole in velvetleaf (Abutilon theophrasti). Journal of AOAC International, 96(1), 1-6. http://dx.doi.org/10.5740/jaoacint.12-166

Gonzalez-Rodriguez, R. M., Rial-Otero, R., Canchogrande, B., \& Simal-Gándara, J. (2011). A review on the fate of pesticides during the processes within the food-production chain. Critical Reviews in Food Science and Nutrition, 51, 99-114. http://dx.doi.org/10.1080/10408390903432625

Guardia-Rubio, M., Marchal-López, R. M., Ayora-Canada, M. J., \& Ruiz-Medina, A. (2007). Determination of pesticides in olives by gas chromatography using different detection systems. Journal of Chromatography A, 1145, 195-203. http://dx.doi.org/10.1016/j.chroma.2007.01.068

Kin, C. M., \& Huat, T. G. (2010). Headspace solid-phase microextraction for the evaluation of pesticide residue contents in cucumber and strawberry after washing treatment. Food Chemistry, 123, 760-764. http://dx.doi.org/10.1016/j.foodchem.2010.05.038

Li, B. J. (2010). Reflections on Hainan cowpea events. Chinese vegetable, 5, 1-2.

Lin, C. S., Tsai, P. J., Wu, C., Yeh, J. Y., \& Saalia, F. K. (2006). Evaluation of electrolysed water as an agent for reducing methamidophos and dimethoate concentrations in vegetables. International Journal of Food Science and Technology, 41, 1099-1104. http://dx.doi.org/10.1111/j.1365-2621.2006.01192.x

Luís, P. D. M. P., Lourival, C. P., Luiz, L. F., Luiz, R., \& Pimentel, T. (2005). Kinetics of carbosulfan hydrolysis to carbofuran and the subsequent degradation of this last compound in irrigated rice fields. Chemosphere, 60, 149-156. http://dx.doi.org/10.1016/j.chemosphere.2005.02.049

Muhammad, I. T., Shahzad, A., \& Ishtiaq, H. (2006). Degradation and persistence of cotton pesticides in sandy loam soils from Punjab, Pakistan. Environmental Research, 100, 184-196. http://dx.doi.org/10.1016/j.envres.2005.05.002

Pan, Y. F., Luo, F., \& Lei, C. L. (2005). Major pests and natural enemies in cowpea field ecosystem. Chinese Bulletin of Entomology, 42, 404-407.

Radwan, M. A., Abu-Elamayem, M. M., Shiboob, M. H., \& Abdel-Aal, A. (2005). Residual behaviour of profenofos on some field-grown vegetables and its removal using various washing solutions and household 
processing. Food and Chemical Toxicology, 43, 553-557. http://dx.doi.org/10.1016/j.fct.2004.12.009

Rasmussen, R. R., Poulsen, M. E., \& Hansen, H. C. B. (2003). Distribution of multiple pesticide residues in apple segments after home processing. Food Additives and Contaminants, 20, 1044-1063. http://dx.doi.org/10.1080/02652030310001615221

St-amand, A. D., \& Girard, L. (2004). Determination of acephate and its degradation product methamidophos in soil and water by solid-phase extraction (SPE) and GC-MS. International Journal of Environmental and Analytical Chemistry, 84, 739-748. http://dx.doi.org/10.1080/03067310410001729600

Zhang, Z. Y., Liu, X. J., \& Hong, X. Y. (2007). Effects of home preparation on pesticide residues cabbage. Food Control, 8, 1484-1487. http://dx.doi.org/10.1016/j.foodcont.2006.11.002

\section{Copyrights}

Copyright for this article is retained by the author(s), with first publication rights granted to the journal.

This is an open-access article distributed under the terms and conditions of the Creative Commons Attribution license (http://creativecommons.org/licenses/by/3.0/). 